\title{
Evaluación energética comparativa de un sistema híbrido eólico- fotovoltaico de baja potencia para la electrificación de una vivienda rural
}

\section{Comparative energy evaluation of a hybrid wind-photovoltaic system of low power for the electrification of rural housing}

Karina Pacco

Universidad Nacional Jorge Basadre Grohmann

\section{RESUMEN}

El presente trabajo está basado en la tesis titulada: "Evaluación energética comparativa de un Sistema Híbrido Eólico-Fotovoltaico de baja potencia para la electrificación de una vivienda rural" realizada durante el periodo Febrero-Marzo 2010, tiene por finalidad evaluar los recursos eólico y solar de la Provincia de Tacna y evaluar comparativamente un sistema eólico-fotovoltaico con sus sub-sistemas para la electrificación de una vivienda rural ubicada en la Provincia de Tacna. En la investigación se utilizó un aerogenerador Rutland WG910 y un panel fotovoltaico Siemens, se construyeron instrumentos como el divisor de voltaje y el circuito fotoeléctrico; se desarrolló un software-plantilla para el estudio detallado de los recursos solar y eólico, los sistemas energéticos y los datos meteorológicos; así también se muestra los detalles de la tecnología del sistema híbrido eólico-fotovoltaico de forma que el sistema híbrido para una vivienda que se proyecte en el futuro inmediato sea más eficiente y económica.

Los subsistemas que integran el sistema híbrido eólico-fotovoltaico son generadores domésticos de electricidad económicos, que se instalan a bajo costo y ayudan a liberar a los ciudadanos de la dependencia de las compañías de suministro eléctrico y del petróleo. Los resultados obtenidos de la evaluación indican la posibilidad de un uso mayor de la energía solar y la no viabilidad de la energía eólica para la Provincia de Tacna, por lo tanto si se hiciera una reconversión energética hacia energías renovables; la energía solar podría ser el nuevo vector energético sostenible de la Provincia de Tacna para su alto progreso material y su desarrollo sostenible. Así también se analizó la viabilidad técnico-económica de la producción de cada subsistema y se utilizó un método basado en evaluar la fiabilidad de un sistema autónomo mediante el concepto de Probabilidad de Pérdida de Carga, generalmente denominada LLP (Loss of Load Probability).

En la actualidad, a escala mundial, la energía consumida por el hombre proviene de los combustibles fósiles cuya utilización masiva conduce al agotamiento de las reservas y supone a la vez un real peligro para el medio ambiente, manifestándose principalmente a través del calentamiento global de la tierra y la acidificación del ciclo del agua. Es importante hacer la reconversión energética hacia energías independientes del petróleo y limpias para romper la dependencia económica de los proveedores de combustibles fósiles y apostar por un desarrollo sostenible.

El objetivo de la presente investigación es realizar la evaluación comparativa de un sistema híbrido eólicofotovoltaico de baja potencia para la electrificación de una vivienda rural" ubicada en la provincia de Tacna, para ello es necesario analizar los parámetros meteorológicos de la intensidad del viento y la radiación solar local, así como el análisis energético y posibilidades de uso del sistema híbrido eólico-fotovoltaico, considerándose como una alternativa para el sector energético. A lo largo de la recolección de datos y elaboración del informe de tesis, se desarrolló un software para el estudio detallado de los recursos solar y eólico, los sistemas energéticos y los datos meteorológicos.

Descriptores: Shefv, energía eólica, energía solar, software plantilla, software estadístico, LLP, Tacna, divisor de voltaje, circuito fotoeléctrico, detalles tecnológicos, Rutland WG-910, Siemens, recurso eólico, recurso solar. 


\section{ABSTRACT}

This work is based on the thesis titled "Comparative Energy evaluation of a hybrid wind-photovoltaic system of low power for the electrification of rural housing" conducted during February-March 2010, aims to evaluate wind and solar resources of the province of Tacna and benchmark wind-photovoltaic system with its subsystems for the electrification of a rural home located in the province of Tacna. The research used a wind turbine Rutland WG-910 and a Siemens solar panel, built instruments such as the voltage divider and the circuit Photoelectric developed a software template for the detailed study of solar and wind resources, energy systems and meteorological data, and also shows details of the technology of wind-photovoltaic hybrid system so that the hybrid system for housing projected into the near future more efficiently and economically.

The subsystems that make up the hybrid wind-photovoltaic are home generators of economic power that are installed at low cost and help to free the citizens of the dependence of the power companies and oil. The results of the evaluation indicate the possibility of greater use of solar energy and non-viability of wind power for the province of Tacna, so if you make a reconversion to renewable energy, solar energy could be the new sustainable energy vector in the province of Tacna to its high material progress and sustainable development. Thus we also analyzed the technical and economic feasibility of production of each subsystem and used a method based on evaluating the reliability of an autonomous system using the concept of Loss of Load Probability, usually referred LLP.

At present, on a global scale, the power consumed by humans comes from fossil fuels whose massive use leads depletion of reserves and involve both a real danger to the environment, manifesting mainly through the global warming of the earth and the acidification of the water cycle. It is important to energy conversion to energy independent, clean oil to break the economic dependence on suppliers of fossil fuels and go for sustainable development.

The objective of this research is to make a "Comparative Energy evaluation of a hybrid wind-photovoltaic system of low power for the electrification of rural housing" located in the province of Tacna, for it is necessary to analyze the meteorological parameters of wind speed and local solar radiation and energy analysis and possible applications of wind-photovoltaic hybrid system, considered as an alternative to the energy sector. Throughout the collection of data and elaboration of the thesis report, it developed a software detailed study of solar and wind resources, energy systems and meteorological data.

Keywords: Shefv, wind energy, solar energy, template software, statistical software, LLP, Tacna, voltage divider, photoelectric circuit, technology details, Rutland WG-910, Siemens, wind resources, solar resources.

\section{INTRODUCCIÓN}

En nuestro país existen 6 millones 400 mil 131 viviendas particulares con ocupantes presentes de los cuales; 4 millones 741 mil 730 disponen de alumbrado eléctrico conectado a la red pública, mientras que un millón 658 mil 401 viviendas aún no disponen de este servicio (INEI - Censos Nacionales de Población y Vivienda 2007). En cifras relativas, el $74,1 \%$ de las viviendas dispone de este servicio, que significa un importante crecimiento en relación a 1993, que fue de 54,9\%. En cambio, las viviendas que no disponen del servicio de alumbrado eléctrico han disminuido de $45,1 \%$ en 1993 a $25,9 \%$ en el 2007; siendo el principal motivo la orografía tan variada y la dispersión de las poblaciones rurales, haciendo que el costo de tendido de redes eléctricas sea alto, por lo que se hace necesario buscar alternativas de electrificación con modelos diferentes. En la presente investigación se realiza el estudio de los recursos eólico y solar del Distrito de Tacna, con el fin de generar energía eléctrica, para mejorar la calidad de vida de la población del Distrito de Tacna, mostrando los beneficios que se podrían obtener de la energía eólica y solar; y las posibilidades de convertirse en el vector energético sostenible para la Región Tacna.

\section{ANTECEDENTES DE LA INVESTIGACIÓN Vientos de Tacna}

El Informe de prácticas pre-profesionales "Vientos de Tacna", ha sido realizada tomando como base la data aportada de los años 2000-Abril 2005 de la Región Tacna de la Red de Monitoreo SENAMHI Tacna-Moquegua, realizada en el periodo correspondiente de Noviembre 2004 a Agosto 2005.

\section{Atlas de Energía Solar del Perú}

Este libro ha sido realizado tomando como base lo aportado por la base de datos de irradiación solar proveniente de estaciones convencionales y automáticas instaladas a nivel nacional del Servicio Nacional de Meteorología e Hidrología del Perú (SENAMHI), siendo elaborada a través del convenio 
ECIPERÚ

Proyecto PER/98/G31 entre el SENAMHI y la Dirección Ejecutiva de Proyectos del Ministerio de Energía y Minas.

\section{Sistema mixto eólico fotovoltaico en vivienda rural basada en el concepto de "Permacultura"}

Esta tesis doctoral que lleva por nombre Sistema Mixto Eólico-Fotovoltaico SHEFV para una vivienda rural en la localidad de Piña de Esgueva (Valladolid) basada en el concepto de "PERMACULTURA"; tiene por finalidad ofrecer un método de aprovechamiento de los recursos que la naturaleza nos brinda sin por ello tener que causar daños irreversibles que pusieran en peligro la sostenibilidad del entorno, el proyecto tiene un claro carácter multidisciplinar, recabando los conocimientos necesarios en agricultura ecológica, arquitectura bioclimática, tratamiento de residuos y energías renovables.

\section{OBJETIVOS DE TRABAJO}

\section{Objetivo General}

Realizar una evaluación energética comparativa de un Sistema Híbrido Eólico-Fotovoltaico de baja potencia con sus subsistemas autónomos (sistema eólico y sistema fotovoltaico) para la electrificación de una vivienda rural para su reconversión energética.

\section{Objetivos Específicos}

1. Hacer uso de la energía eólica y solar para la utilización eficiente y sostenible de la energía eléctrica para el dimensionamiento de una instalación de un Sistema Híbrido EólicoFotovoltaico de baja potencia para una vivienda rural de la provincia de Tacna.

2. Realizar una evaluación de los recursos eólico y solar en un lugar referencial con fines de extrapolación a zonas con características similares en recursos eólico y solar.

3. Evaluar la calidad final del Sistema Híbrido Eólico-Fotovoltaico de baja potencia a través de la eficiencia energética, correcto dimensionamiento, servicio de energía eléctrica e instalación.

\section{FORMULACIÓN DE HIPÓTESIS}

\section{Hipótesis General}

La evaluación energética comparativa de un Sistema Híbrido Eólico-Fotovoltaico de baja potencia con sus subsistemas autónomos (sistema eólico y sistema fotovoltaico) para la electrificación de una vivienda rural, es factible desde el punto de vista económico.

\section{Hipótesis Específicas}

1. Es posible, hacer uso de la energía eólica y solar para la utilización eficiente y sostenible de la energía para el dimensionamiento de una instalación de un Sistema Híbrido EólicoFotovoltaico de baja potencia para una vivienda rural de la provincia de Tacna para su reconversión energética.

2. La evaluación y el análisis de las condiciones meteorológicas y climatológicas del lugar referencial son importantes para determinar la eficiencia del Sistema Híbrido Eólico-Fotovoltaico de baja potencia.

3. Es posible, realizar la evaluación de la calidad final del Sistema Híbrido Eólico-Fotovoltaico de baja potencia indicando el suministro de electricidad y significando que se evitará la emisión a la atmósfera de dióxido de carbono (CO2) y dióxido de azufre (SO2).

\section{CONTRIBUCIONES DEL AUTOR}

1. Mostrar los detalles de la tecnología del sistema híbrido eólico-fotovoltaico para una vivienda.

2. Desarrollar un software para el estudio detallado de los recursos solar y eólico, los sistemas energéticos y los datos meteorológicos.

3. Realizó estudios sistemáticos de confiabilidad en tecnología y aplicación, la electrificación híbrida eólica-fotovoltaica. Mediante el concepto de Probabilidad de Pérdida de Carga, generalmente denominada LLP (Loss of Load Probability), tratando de forma conjunta la fiabilidad asociada al dimensionado de las instalaciones.

\section{EXPERIMENTAL}

\section{MATERIAL Y MÉTODOS}

Unidades de Análisis: En el estudio se utilizaron: 1 aerogenerador Rutland 910, 1 panel fotovoltaico Siemens, 1 divisor de voltaje, 1 circuito fotoeléctrico, 1 datalogger; y programas como: (Beta) Shefv Studio para el estudio de los recursos eólico y solar para los datos recopilados del periodo Febrero-Marzo 2010,

WrPlot View versión 5.9, Weatherlink 5.5, Statgraphics Centurion XVI y SPSS versión 18.

\section{Metodología:}

Para la fase experimental se trabajó con los dos sistemas con conexión en paralelo, conectados al datalogger (previamente ambos sistemas estaban conectados a un divisor de voltaje), para realizar la evaluación se midió el voltaje de los sistemas en 
paralelo y los datos meteorológicos (eólico y solar) las 24 horas del día de lunes a domingo. Para la obtención de los datos del squirrel se uso el programa de Análisis de Squirrel y de la estación meteorológica Vantage Pro2 el programa Weatherlink 5.5.1.

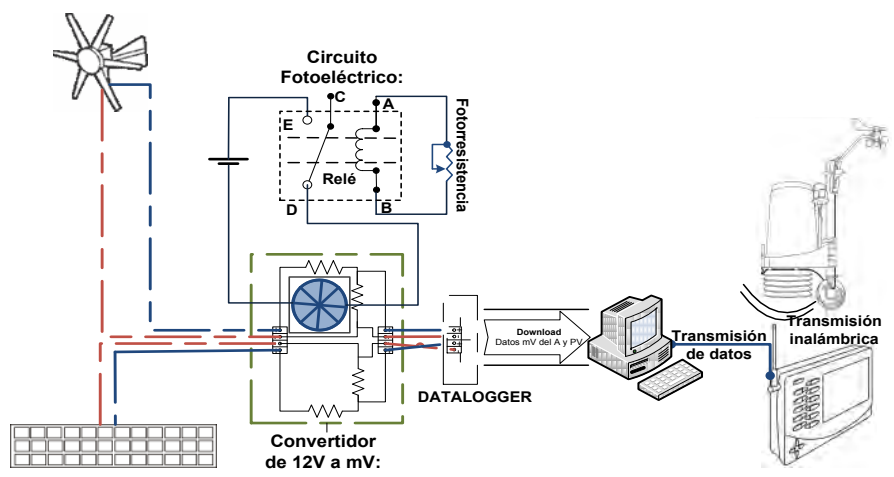

Gráfico 1. Esquema de instalación de los diversos equipos

Se procesaron los datos haciendo uso de los programas como Statgraphics Centurión, SPSS, WrPlot View, Shefv Studio (Beta) entre otros, obteniendo las curvas respectivas las cuales fueron utilizadas para la evaluación energética comparativa y dimensionamiento del sistema híbrido eólico fotovoltaico para una vivienda rural. Se calculó la eficiencia del sistema para conocer su rentabilidad en una vivienda rural.

\section{RESULTADOS Y DISCUSIÓN}

Tabla №01. Datos eólicos diarios del distrito de Tacna para el periodo febrero-marzo 2010

Estación: Centro de Energías Renovables de Tacna-CERT

\begin{tabular}{|c|c|c|c|c|c|c|c|}
\hline $\begin{array}{c}\text { Día Ordinal } \\
{[-]}\end{array}$ & $\begin{array}{c}\text { Velocidad } \\
{[\mathrm{m} / \mathrm{s}]}\end{array}$ & $\begin{array}{c}\text { Dirección } \\
{[-]}\end{array}$ & 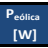 & $\begin{array}{c}\text { Día Ordinal } \\
{[-]}\end{array}$ & $\begin{array}{c}\text { Velocidad } \\
{[\mathrm{m} / \mathrm{s}]}\end{array}$ & $\begin{array}{c}\text { Dirección } \\
{[-]}\end{array}$ & $\begin{array}{l}\begin{array}{c}\text { Pesiles } \\
\text { [W] }\end{array} \\
\end{array}$ \\
\hline 32 & 2.06 & NW & 3.35 & 62 & 1.94 & $\mathrm{~N} / \mathrm{NE}$ & 2.82 \\
\hline 33 & 1.94 & NNW & 2.83 & 63 & 2.21 & NE & 4.18 \\
\hline 34 & 1.78 & NNW & 2.19 & 64 & 2.11 & NE & 3.64 \\
\hline 35 & 2.05 & $\mathrm{NE}$ & 3.31 & 65 & 2.19 & NE & 4.07 \\
\hline 36 & 2.27 & $\mathrm{NE}$ & 4.50 & 66 & 2.26 & $\mathrm{NE}$ & 4.43 \\
\hline 37 & 1.77 & NNE & 2.12 & 67 & 2.13 & $\mathrm{NE}$ & 3.71 \\
\hline 38 & 2.14 & $\mathrm{NE}$ & 3.77 & 68 & 2.06 & NE & 3.39 \\
\hline 39 & 1.87 & NE & 2.53 & 69 & 2.36 & $\mathrm{~N}$ & 5.08 \\
\hline 40 & 2.03 & NNW & 3.23 & 70 & 2.18 & NE & 4.02 \\
\hline 41 & 1.73 & NNW & 1.99 & 71 & 2.45 & $\mathrm{~N}$ & 5.71 \\
\hline 42 & 2.35 & $\mathrm{NE}$ & 5.02 & 72 & 2.42 & NNE & 5.46 \\
\hline 43 & 2.27 & NE & 4.50 & 73 & 2.29 & $\mathrm{~N}$ & 4.65 \\
\hline 44 & 2.24 & NE & 4.33 & 74 & 2.24 & NE & 4.34 \\
\hline 45 & 2.14 & $\mathrm{NE}$ & 3.77 & 75 & 2.46 & $\mathrm{NE}$ & 5.71 \\
\hline 46 & $\begin{array}{l}2.14 \\
2.32\end{array}$ & $\begin{array}{l}\text { Ne } \\
\text { NNE }\end{array}$ & 4.79 & 76 & $\begin{array}{l}2.406 \\
2.23\end{array}$ & $\begin{array}{l}\text { NE } \\
\text { NNE }\end{array}$ & $\begin{array}{l}4.71 \\
4.27\end{array}$ \\
\hline 47 & 2.15 & NNE & 3.81 & 77 & 2.44 & $\mathrm{NE}$ & 5.63 \\
\hline 48 & 2.53 & $\mathrm{NE}$ & 6.25 & 78 & 2.59 & $\mathrm{~N}$ & 6.73 \\
\hline 49 & 2.39 & NNE & 5.25 & 79 & 2.39 & NNE & 5.28 \\
\hline 50 & 2.60 & $\mathrm{NE}$ & 6.80 & 80 & 2.39 & $\mathrm{NE}$ & 5.27 \\
\hline 51 & 2.04 & NNE & 3.30 & 81 & 1.72 & $\mathrm{NE}$ & 1.97 \\
\hline 52 & $\begin{array}{l}2.049 \\
2.39\end{array}$ & $\mathrm{NE}$ & 5.29 & 82 & $\begin{array}{l}2.07 \\
2.07\end{array}$ & $\mathrm{~N}$ & 3.43 \\
\hline 53 & 2.75 & $\mathrm{NE}$ & 8.01 & 83 & 2.51 & NE & 6.10 \\
\hline 54 & 2.12 & $\mathrm{NE}$ & 3.70 & 84 & 2.25 & $\mathrm{NE}$ & 4.37 \\
\hline 55 & 2.45 & $\mathrm{NE}$ & 5.67 & 85 & 2.20 & NE & 4.13 \\
\hline 56 & 2.27 & $\mathrm{NE}$ & 4.50 & 86 & 2.03 & NE & 3.22 \\
\hline 57 & 2.30 & NE & 4.71 & 87 & 1.86 & $\mathrm{NE}$ & 2.48 \\
\hline 58 & 2.56 & NNE & 6.46 & 88 & 2.13 & $\mathrm{NE}$ & 3.72 \\
\hline 59 & 2.36 & $\mathrm{NE}$ & $\begin{array}{l}5.400 \\
5.07\end{array}$ & $\begin{array}{l}80 \\
89\end{array}$ & 2.24 & $\mathrm{NE}$ & 4.36 \\
\hline 60 & 2.47 & $\mathrm{~N}$ & 5.81 & 90 & 2.19 & $\mathrm{NE}$ & 4.03 \\
\hline 61 & 2.88 & NNE & 9.22 & & & & \\
\hline
\end{tabular}

Estación Meteorológica: Centro de Energías Renovables de Tacna-CERT.

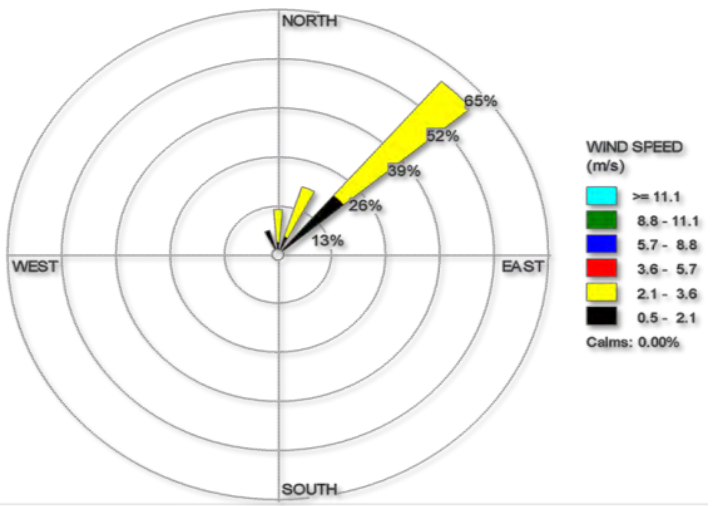

Gráfico №02. Rosa de los vientos para el periodo febrero-marzo 2010

\begin{tabular}{lcll}
\multicolumn{4}{c}{ Análisis del comportamiento } \\
№ de días del mes >=2 m/s: & 51 & días \\
№ Horas del mes >=2m/s: & 344.47 & horas \\
Prom. de Velocidad del periodo: & 2.23 & $\mathrm{~m} / \mathrm{s}$ \\
Vel. mínima del periodo: & 1.72 & $\mathrm{~m} / \mathrm{s}$ \\
Vel. máxima del periodo: & 2.88 & $\mathrm{~m} / \mathrm{s}$ \\
Direc. predominante del periodo: & $\mathrm{NE}$ & \\
\% de calma del viento: & 0.00 & $\%$ \\
Fuente: Tabla №01 & &
\end{tabular}

Cuadro №01. Comparación del número de horas de velocidad de viento de los meses de febrero y marzo y horas totales para el periodo febrero-marzo 2010

Estación Meteorológica: Centro de Energías Renovables de TacnaCERT

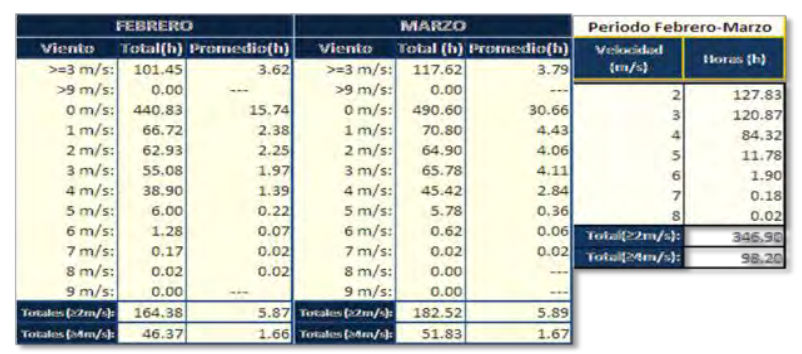

Tabla №02. Datos energéticos diarios del SME para el periodo febrero-marzo 2010

Estación: Centro de Energías Renovables de Tacna- CERT 


\begin{tabular}{|c|c|c|c|c|c|c|c|c|c|}
\hline $\begin{array}{c}\text { Día } \\
\text { Ordinal }\end{array}$ & $\begin{array}{c}\text { I Aero } \\
{[\text { A] }}\end{array}$ & $\begin{array}{c}\begin{array}{c}\text { V Aero } \\
\text { [V] }\end{array} \\
\end{array}$ & $\begin{array}{c}\mathbf{P}_{\mathrm{p}} \text { Aero } \\
\text { [W] }\end{array}$ & $\begin{array}{c}\begin{array}{c}\text { P Aero } \\
\text { [kW] }\end{array} \\
\end{array}$ & $\begin{array}{c}\text { Día } \\
\text { Ordinal }\end{array}$ & $\begin{array}{c}\text { I Aero } \\
{[\mathrm{A}]}\end{array}$ & $\begin{array}{c}\begin{array}{c}\text { V Aero } \\
\text { [V] }\end{array} \\
\end{array}$ & $\begin{array}{c}P_{p} \text { Aero } \\
{[W]}\end{array}$ & $\begin{array}{l}\text { P Aero } \\
{[\mathrm{kW}]}\end{array}$ \\
\hline 32 & 0.37 & 1.97 & 1.62 & 0.38 & 62 & 0.25 & 1.33 & 0.99 & 0.26 \\
\hline 33 & 0.39 & 2.02 & 1.65 & 0.41 & 63 & 0.32 & 1.70 & 1.31 & 0.44 \\
\hline 34 & 0.30 & 1.59 & 1.24 & 0.23 & 64 & 0.31 & 1.61 & 1.13 & 0.35 \\
\hline 35 & 0.27 & 1.42 & 0.93 & 0.26 & 65 & 0.29 & 1.51 & 1.12 & 0.38 \\
\hline 36 & 0.33 & 1.75 & 1.36 & 0.53 & 66 & 0.32 & 1.66 & 1.28 & 0.41 \\
\hline 37 & 0.50 & 2.60 & 2.05 & 0.41 & 67 & 0.32 & 1.68 & 1.34 & 0.39 \\
\hline 38 & 0.30 & 1.60 & 1.17 & 0.42 & 68 & 0.27 & 1.43 & 1.06 & 0.29 \\
\hline 39 & 0.26 & 1.35 & 0.96 & 0.30 & 69 & 0.25 & 1.32 & 0.97 & 0.33 \\
\hline 40 & 0.27 & 1.41 & 0.99 & 0.36 & 70 & 0.28 & 1.48 & 1.12 & 0.35 \\
\hline 41 & 0.18 & 0.95 & 0.56 & 0.14 & 71 & 0.27 & 1.42 & 1.00 & 0.36 \\
\hline 42 & 0.22 & 1.17 & 0.72 & 0.30 & 72 & 0.31 & 1.61 & 1.22 & 0.38 \\
\hline 43 & 0.33 & 1.75 & 1.36 & 0.53 & 73 & 0.31 & 1.60 & 1.20 & 0.36 \\
\hline 44 & 0.30 & 1.59 & 1.18 & 0.45 & 74 & 0.28 & 1.48 & 1.06 & 0.33 \\
\hline 45 & 0.30 & 1.60 & 1.17 & 0.42 & 75 & 0.29 & 1.50 & 1.12 & 0.41 \\
\hline 46 & 0.33 & 1.71 & 1.24 & 0.50 & 76 & 0.30 & 1.57 & 1.10 & 0.28 \\
\hline 47 & 0.34 & 1.78 & 1.31 & 0.39 & 77 & 0.30 & 1.56 & 1.12 & 0.38 \\
\hline 48 & 0.37 & 1.94 & 1.48 & 0.62 & 78 & 0.33 & 1.74 & 1.30 & 0.43 \\
\hline 49 & 0.40 & 2.11 & 1.63 & 0.66 & 79 & 0.30 & 1.59 & 1.17 & 0.40 \\
\hline 50 & 0.35 & 1.85 & 1.39 & 0.68 & 80 & 0.31 & 1.60 & 1.19 & 0.37 \\
\hline 51 & 0.35 & 1.83 & 1.40 & 0.45 & 81 & 0.32 & 1.71 & 1.17 & 0.11 \\
\hline 52 & 0.43 & 2.27 & 1.66 & 0.53 & 82 & 0.33 & 1.73 & 1.30 & 0.40 \\
\hline 53 & 0.41 & 2.15 & 1.90 & 0.72 & 83 & 0.28 & 1.47 & 1.00 & 0.42 \\
\hline 54 & 0.36 & 1.91 & 1.59 & 0.50 & 84 & 0.26 & 1.39 & 0.99 & 0.49 \\
\hline 55 & 0.33 & 1.75 & 1.39 & 0.60 & 85 & 0.28 & 1.44 & 1.01 & 0.50 \\
\hline 56 & 0.31 & 1.65 & 1.30 & 0.49 & 86 & 0.27 & 1.40 & 0.98 & 0.47 \\
\hline 57 & 0.32 & 1.70 & 1.40 & 0.58 & 87 & 0.24 & 1.27 & 0.83 & 0.35 \\
\hline 58 & 0.31 & 1.63 & 1.27 & 0.62 & 88 & 0.24 & 1.28 & 0.83 & 0.38 \\
\hline 59 & 0.28 & 1.49 & 1.08 & 0.44 & 89 & 0.25 & 1.31 & 0.87 & 0.36 \\
\hline 60 & 0.35 & 1.84 & 1.50 & 0.59 & 90 & 0.25 & 1.29 & 0.87 & 0.28 \\
\hline 61 & 0.34 & 1.77 & 1.40 & 0.70 & & & & & \\
\hline
\end{tabular}

Tabla №03. Datos de potencia eléctrica de salida del aerogenerador Rutland wg-910 y número de horas para el periodo febrero-marzo 2010

Estación Meteorológica: Centro de Energías Renovables de TacnaCERT

\begin{tabular}{|c|c|c|c|c|}
\hline \multicolumn{3}{|c|}{ ESTIMACIÓN DE ENERGÍA DEL AEROGENERADOR: } & \multicolumn{2}{|c|}{$\begin{array}{c}\text { Rutland WG-910 } \\
76 \mathrm{~W}\end{array}$} \\
\hline $\begin{array}{l}\text { Velocidad } \\
(\mathrm{m} / \mathrm{s})\end{array}$ & Potencia (W) & Potencia $_{\text {norm }}(-)$ & Horas (h) & $\begin{array}{l}\text { Producción } \\
\text { (kWh) }\end{array}$ \\
\hline 2 & 0 & 0.00 & 127.83 & 0.00 \\
\hline 3 & 6 & 0.08 & 120.87 & 0.73 \\
\hline 4 & 10 & 0.13 & 84.32 & 0.84 \\
\hline 5 & 14 & 0.18 & 11.78 & 0.16 \\
\hline 6 & 25 & 0.33 & 1.90 & 0.05 \\
\hline 7 & 31 & 0.41 & 0.18 & 0.01 \\
\hline 8 & 39 & 0.51 & 0.02 & 0.00 \\
\hline 9 & 47 & 0.62 & 0.00 & 0.00 \\
\hline 9.6 & 56 & 0.74 & 0.0 & 0.00 \\
\hline 10 & 65 & 0.86 & 0.0 & 0.00 \\
\hline 11 & 76 & 1.00 & 0.0 & 0.00 \\
\hline 12 & 87 & 1.14 & 0.0 & 0.00 \\
\hline 13 & 99 & 1.30 & 0.0 & 0.00 \\
\hline 13.5 & 112 & 1.47 & 0.0 & 0.00 \\
\hline 14 & 125 & 1.64 & 0.0 & 0.00 \\
\hline 15 & 140 & 1.84 & 0.0 & 0.00 \\
\hline 16 & 146 & 1.92 & 0.0 & 0.00 \\
\hline 17 & 158 & 2.08 & 0.0 & 0.00 \\
\hline 18 & 158 & 2.08 & 0.0 & 0.00 \\
\hline 19 & 159 & 2.09 & 0.0 & 0.00 \\
\hline 20 & 162 & 2.13 & 0.0 & 0.00 \\
\hline & & TOTAL: & 346.9 & 1.8 \\
\hline
\end{tabular}

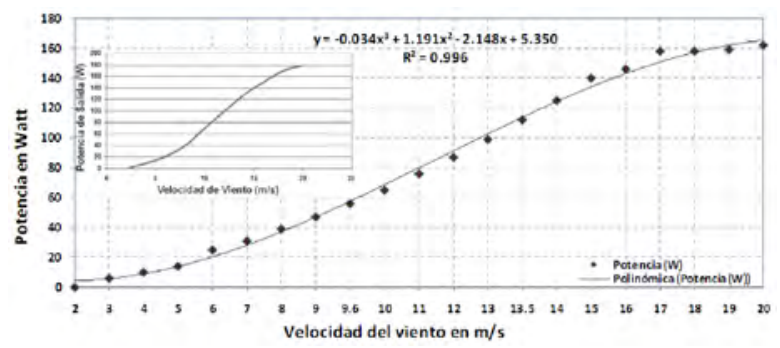

Gráfico №03. Curva de potencia del aerogenerador rutland wg-

\section{Comentario}

La tabla №03 muestra los valores de velocidad, potencia eléctrica de salida (proporcionada por el Dr. Daniel Ferreira de la Universidade Federal de Pernambuco), potencia normalizada y horas del SME obtenidas para el periodo de Febrero-Marzo del 2010. Los valores de la tabla №03 están bien representados por la curva esquematizada en el gráfico №16 para el aerogenerador Rutland WG-910 de la marca Marlec; el valor de la velocidad máxima es $20 \mathrm{~m} / \mathrm{s}$, su potencia eléctrica es $159 \mathrm{~W}$ y la velocidad mínima es $2 \mathrm{~m} / \mathrm{s}$, su potencia eléctrica es OW.

El gráfico №03 presenta la Curva de potencia característica del Aerogenerador Rutland WG-910 de la marca Marlec (indica cuál será la potencia eléctrica disponible en el aerogenerador para diferentes velocidades de viento). La curva de potencia es válida para las siguientes condiciones estándar del aire: densidad de aire de $1.225 \mathrm{~kg} / \mathrm{m}^{3}$, palas de rotor limpias y flujo de aire horizontal no perturbado. En esta gráfica se puede observar del comportamiento de la curva representativa, que la producción de potencia eléctrica disponible es directamente proporcional a la velocidad del viento $(P \sim v)$. El gráfico №03, muestra la línea de tendencia (o regresión) de la potencia eléctrica disponible y la velocidad del viento, representada en la ecuación $Y$ $=-0.034 x^{3}+1.191 x^{2}-2.148 x+5.350$, la cual nos ayuda a obtener los valores de potencia eléctrica disponible teniendo como referencia los valores de la velocidad de viento. Esta ecuación es válida para una serie de aerogeneradores de la misma familia Rutland WG-910 de la marca Marlec. El valor $r=1$, nos indica correlación perfecta es decir tendencia a incrementarse la producción de potencia eléctrica disponible con el aumento de la velocidad del viento; y el elevado valor indica "correlación alta positiva", es decir demuestra que la influencia de la velocidad del viento en la producción de potencia eléctrica disponible es importante. Se puede afirmar que el $99.6 \%\left(R^{2}\right)$ de los datos caen sobre la línea de regresión, puede predecirse perfectamente los valores promedios de potencia eléctrica disponible a partir de los valores de la velocidad del viento. 
Estación Meteorológica: Centro de Energías Renovables de Tacna-CERT

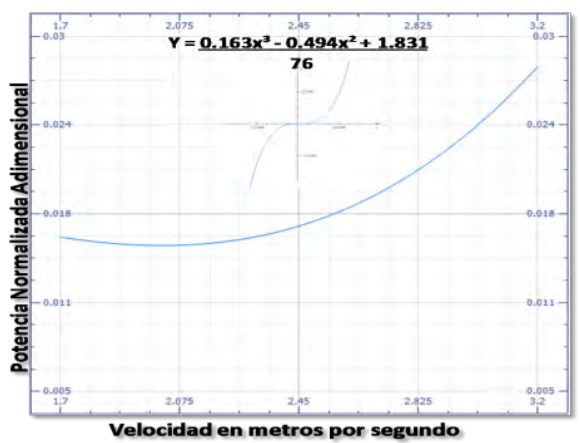

Gráfico №04. Curva de la potencia normalizada del aerogenerador Rutland wg-910 para el periodo febrero-marzo 2010

\section{Comentario}

El gráfico №04 presenta la Curva de la potencia normalizada para el Aerogenerador Rutland WG-910 de la marca Marlec (indica cuál será la potencia normalizada disponible en el aerogenerador para diferentes velocidades de viento) para el periodo Febrero-Marzo. La curva de potencia normalizada representativa se encuentra en el intervalo: $[1.7 \mathrm{~m} / \mathrm{s}-$ $3.2 \mathrm{~m} / \mathrm{s}$ ]. El gráfico №04, muestra la ecuación de la curva de potencia normalizada ploteada, representada por la ecuación $Y=\left(0.163 x^{3}-0.494 x^{2}\right.$ $+1.831) / 76$, la cual nos ayuda a obtener los valores de potencia normalizada teniendo como referencia los valores de la velocidad de viento. Esta ecuación es válida para una serie de aerogeneradores de la misma familia Rutland WG-910 de la marca Marlec.

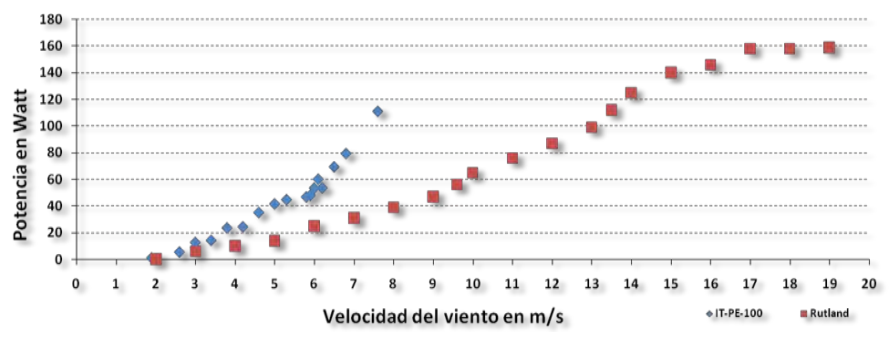

Gráfico №05. Comparación de curvas de potencia de los aerogeneradores it-pe-100 y Rutland wg-910.

\section{Comentario}

IT-PE-100 versus Rutland WG-910 (Gráfico №05) Los aerogeneradores comparados de aproximación polinómica de orden cuatro de la relación experimental entre potencia y velocidad del viento válida en el intervalo $v \in[0, \ldots 19]$.

$$
\sum_{i=0}^{4} a_{i} x_{i}
$$

De la representación de las funciones $\mathrm{P}(\mathrm{v}) \mathrm{IT}-\mathrm{PE}-100$ y $P(v) W G-910$ en un gráfico conjunto se obtienen las siguientes conclusiones: El aerogenerador IT-PE-100 trabaja con velocidades del viento bajas, en el intervalo $\vee \in[1.9, \ldots$ 7.6]. $Y$ el aerogenerador Rutland WG-910 trabaja con velocidades del viento altas, en el intervalo $\vee \in[2, \ldots 20]$. Por lo tanto el aerogenerador IT-PE-100 trabaja en un rango de velocidades menor que el aerogenerador Rutland WG-910. El aerogenerador IT-PE-100 tiene una potencia de salida mayor que el Rutland WG-910. Para el margen de velocidades comprendido entre 3 y $9 \mathrm{~m} / \mathrm{s}$.

Tabla №04. Datos diarios de radiación solar global horizontal diaria para el distrito de Tacna periodo febrero-marzo 2010 Estación: Centro de Energías Renovables de Tacna-CERT

\begin{tabular}{|c|c|c|c|c|c|c|c|c|c|}
\hline Dia & | Irradiancie & radiacic & Rad_sola & Rad_sola & $\begin{array}{c}\text { Dia } \\
\text { Ordina }\end{array}$ & |rradiancia & Irradiación & ad_sola & Iad_sola \\
\hline & {$\left[\frac{\mathrm{tkWh} h \mathrm{hm} \cdot \mathrm{d} \cdot \mathrm{d}}{6.19}\right.$} & $\frac{[\mathrm{LWW} / \mathrm{m}}{1.20}$ & & $[\mathrm{~W} / \mathrm{m}] \mathrm{s}]$ & \begin{tabular}{|l|} 
Ordinal \\
62
\end{tabular} & {$[\mathrm{knWW} / \mathrm{m} \cdot \mathrm{d}]$} & {$\left[\begin{array}{ll}{[\mathrm{kWh} / \mathrm{m}]} \\
1.23]\end{array}\right.$} & {$\left[\frac{W\left(m^{2}\right]^{2}}{917}\right.$} & $\frac{\left[W / m^{m}\right]}{585}$ \\
\hline $\begin{array}{l}33 \\
34\end{array}$ & $\begin{array}{l}6.78 \\
6.78\end{array}$ & $\begin{array}{l}1.31 \\
1.13\end{array}$ & $\begin{array}{l}0.97 \\
0.99\end{array}$ & $\begin{array}{l}655 \\
{ }_{5753}\end{array}$ & \begin{tabular}{|l}
63 \\
664
\end{tabular} & $\begin{array}{l}6.53 \\
6.54\end{array}$ & $\begin{array}{l}1.26 \\
1.19\end{array}$ & $\begin{array}{l}903 \\
9017\end{array}$ & $\begin{array}{l}621 \\
5888\end{array}$ \\
\hline 34 & 5.38 & 1.04 & 1.09 & 573 & 64 & 6.11 & 1.19 & 917 & 588 \\
\hline 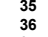 & 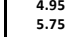 & $\begin{array}{l}0.096 \\
1.11\end{array}$ & $\begin{array}{l}\text { l.31 } \\
1.12\end{array}$ & $\begin{array}{l}4711 \\
590\end{array}$ & $\begin{array}{l}65 \\
66 \\
66\end{array}$ & $\begin{array}{l}6.18 \\
6.31 \\
6.18\end{array}$ & $\begin{array}{l}1.20 \\
1.22\end{array}$ & $\begin{array}{l}899 \\
889\end{array}$ & $\begin{array}{l}619 \\
641 \\
641\end{array}$ \\
\hline $\begin{array}{l}37 \\
38\end{array}$ & 7.11 & $\begin{array}{l}1.38 \\
1.29\end{array}$ & 1.17 & 682 & 67 & $\begin{array}{l}6.41 \\
6.535\end{array}$ & $\begin{array}{l}1.24 \\
1.23\end{array}$ & 894 & 596 \\
\hline & $\begin{array}{l}6.17 \\
6.17\end{array}$ & $\begin{array}{l}1.29 \\
1.20\end{array}$ & & & & $\begin{array}{l}6.35 \\
6.29\end{array}$ & $\begin{array}{l}1.23 \\
1.22\end{array}$ & & $\begin{array}{l}\begin{array}{l}100 \\
600\end{array}\end{array}$ \\
\hline & 5.75 & 1.11 & 0.98 & 586 & 70 & 6.23 & 1.22 & 874 & 597 \\
\hline & 2.58 & 0.50 & 0.49 & 271 & 71 & 6.21 & 1.20 & & 595 \\
\hline & $\begin{array}{l}6.82 \\
6\end{array}$ & 1.13 & 0 & 6 & 73 & $\begin{array}{l}0.26 \\
6.1 .8\end{array}$ & 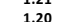 & 8 & $\begin{array}{l}602 \\
599 \\
599\end{array}$ \\
\hline & 6.33 & 1.23 & & 649 & 74 & 6.26 & 1.21 & & 592 \\
\hline 45 & 5.35 & 1.04 & 0.93 & 569 & 75 & 6.21 & 1.20 & 880 & 605 \\
\hline 47 & 5.98 & $\begin{array}{ll}1.1 \\
0\end{array}$ & 0.95 & $\begin{array}{c}629 \\
51\end{array}$ & 77 & $\begin{array}{l}3.39 \\
5\end{array}$ & 0.06 & & $\begin{array}{r}338 \\
575\end{array}$ \\
\hline & 6.89 & 1.34 & 1.09 & 629 & 78 & 5.75 & 1.12 & 860 & $\begin{array}{l}575 \\
581\end{array}$ \\
\hline 49 & 6.25 & 1.21 & 1.14 & 608 & 79 & 5.92 & 1.15 & 855 & 584 \\
\hline & $\begin{array}{r}6.10 \\
{ }^{2}\end{array}$ & $\begin{array}{l}1.18 \\
\end{array}$ & 0.92 & $\begin{array}{l}602 \\
629\end{array}$ & 80 & 5.19 & 1.01 & 880 & 541 \\
\hline & 4.42 & 0.86 & & & 82 & $\begin{array}{l}6.01 \\
6.01\end{array}$ & $\begin{array}{l}1.1 .16 \\
.\end{array}$ & 88 & $\begin{array}{l}273 \\
595 \\
595\end{array}$ \\
\hline & 5.68 & 1.10 & 0.92 & 534 & 83 & 6.08 & 1.18 & 882 & 621 \\
\hline & 5.77 & 1.12 & 0.91 & 566 & 84 & 5.65 & 1.110 & 869 & 618 \\
\hline & . & 1.18 & 0.98 & 5 & 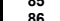 & $\begin{array}{r}3.06 \\
5\end{array}$ & 1.10 & 866 & 602 \\
\hline 57 & 6.66 & 1.29 & 0.91 & 651 & 87 & 5.09 & 0.99 & 989 & $\begin{array}{l}559 \\
559\end{array}$ \\
\hline & $\begin{array}{l}0.02 \\
6.16 \\
6.16\end{array}$ & 1.19 & $\begin{array}{l}0.91 \\
0.97\end{array}$ & $\begin{array}{l}688 \\
588\end{array}$ & $\begin{array}{l}80 \\
89\end{array}$ & 5.90 & $\begin{array}{l}0.199 \\
1.14\end{array}$ & & $\begin{array}{l}547 \\
589\end{array}$ \\
\hline & 6.58 & 1.28 & 908 & 607 & 90 & 5.86 & 1.1 .13 & 869 & 579 \\
\hline
\end{tabular}

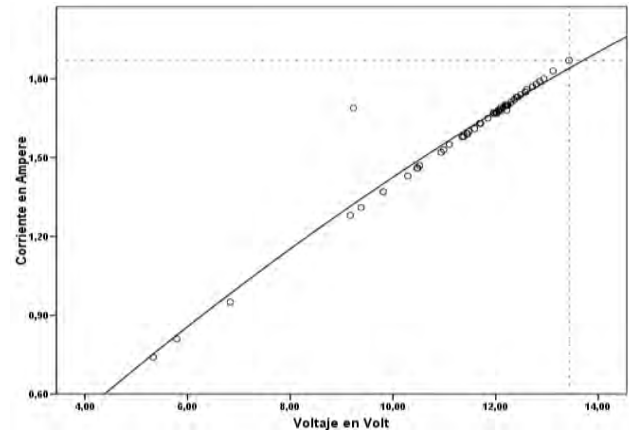

Gráfico №06. Curva de corriente y voltaje influenciada por la radiación promedio del SMFV para el periodo febrero-marzo 2010

\section{Comentario}

El gráfico №60, muestra la línea de tendencia (o regresión) de la corriente y el voltaje para el periodo Febrero-Marzo 2010, representada en la ecuación Y $=-0.003 x^{2}+0.188 x-0.170$, la cual nos ayuda $a$ obtener los valores de corriente teniendo como referencia los valores del voltaje. Esta ecuación es válida para una serie de paneles fotovoltaicos de la misma familia SM-50 de la marca Siemens. El valor $r$ $=0.98$, nos indica correlación directa es decir tendencia a incrementarse la producción de corriente con el aumento; y el elevado valor indica "correlación alta positiva", es decir demuestra que la influencia del voltaje en la producción diaria de corriente para el periodo Febrero-Marzo 2010 es importante. 
Tabla №05. Datos energérticos diarios del SMEFV para el periodo febrero-marzo 2010.

Estación Meteorológica: Centro de Energías Renovables de Tacna-

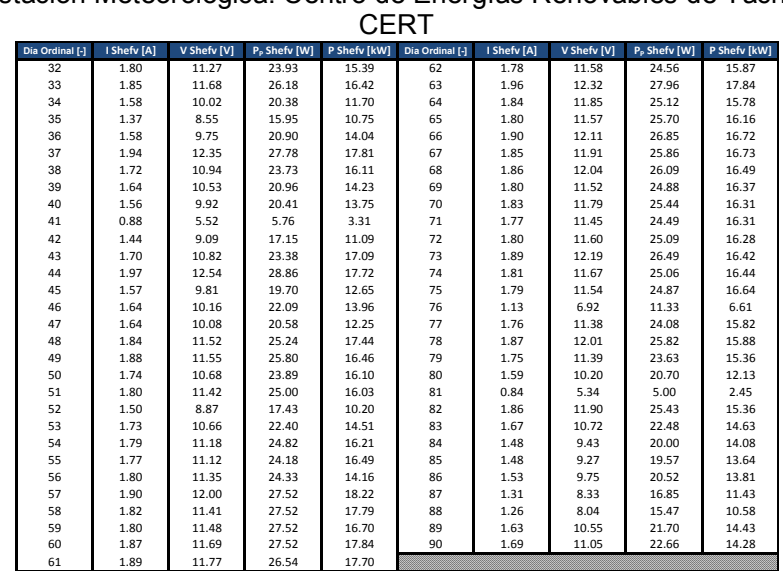

Estación Meteorológica: Centro de Energías Renovables de TacnaCERT

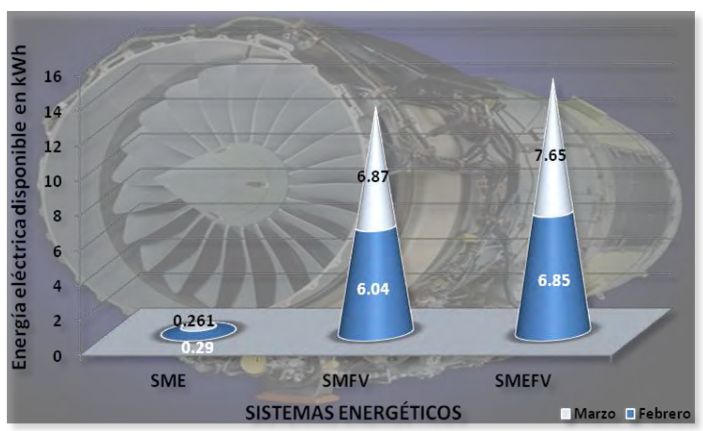

Gráfico №07. Comparación de producción de energía eléctrica obtenida de los SME, SMFV y SMEFV para el periodo febreromarzo 2010

\section{Comentario}

El gráfico №07 muestra la comparación de los tres sistemas con sus respectivas producciones mensuales de energía eléctrica obtenidas durante el periodo Febrero-Marzo 2010, destacando el Shefv seguido del sistema fotovoltaico y con una baja producción el sistema eólico; siendo sus valores: $14.5,12.91$ y $0.55 \mathrm{kWh}$ respectivamente. El gráfico №09 muestra el porcentaje de aporte de energía eléctrica al Shefv de los sistemas solar y eólico, se puede observar que para el periodo Febrero-Marzo 2010 el porcentaje de aportación promedio del sistema eólico es $4 \%$ y del sistema fotovoltaico aportó $96 \%$. Es bastante la diferencia esto se debe al aerogenerador, es un aerogenerador que no trabaja a velocidades de vientos bajos.

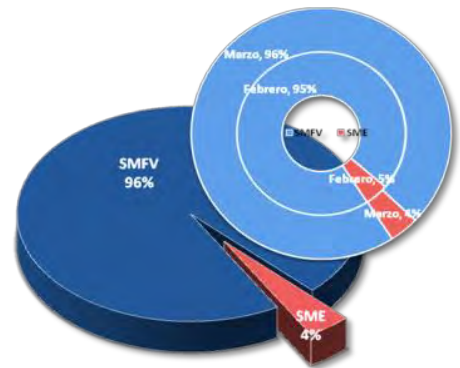

Gráfico №08. Porcentaje de aporte de energía eléctrica al SHEFV obtenida de los sistemas eólico y fotovoltaico para el periodo febrero-marzo 2010

Cuadro №02. Resumen del método de Langer-Beyer (método del peor mes) para el sistema híbrido eólico fotovoltaico para el periodo febrero-marzo 2010

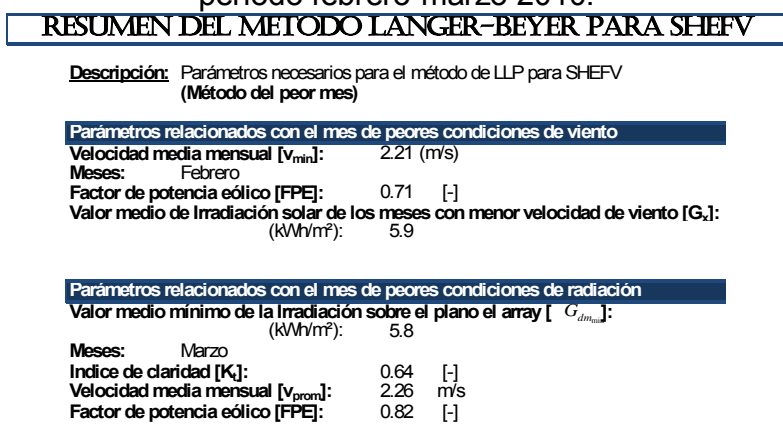

\section{Comentario}

El cuadro №02 muestra el resumen del método de LANGER-BEYER para SHEFV, éste método es parecido al "método del peor mes" del SMFV, se realiza un balance de energía seleccionando un periodo de tiempo, normalmente un mes, el cuál tiene las condiciones más desfavorables de los sistemas eólico y solar (peores condiciones de viento y peores condiciones de radiación), si el sistema funciona en este mes, funcionará también para los demás meses del año. Los resultados que muestra el método de LANGER-BEYER para SHEFV son:

1. El mes con peores condiciones de viento es:

Febrero, con una velocidad media mensual: $2.21 \mathrm{~m} / \mathrm{s}$, con factor de potencia eólico: $0.71 \mathrm{e}$ irradiación media mensual: $5.9 \mathrm{kWh} / \mathrm{m}^{2}$.

2. El mes con peores condiciones de radiación es: Marzo, con una irradiación media mensual: $5.89 \mathrm{kWh} / \mathrm{m}^{2}$, con índice de claridad: 0.64 , con velocidad media mensual: $2.26 \mathrm{~m} / \mathrm{s}$ y con factor de potencia eólico: 0.82 .

No se puede determinar cuál de los dos meses tiene las peores condiciones, el método es para un año, por ello muestra dos resultados diferentes, dos meses diferentes. 


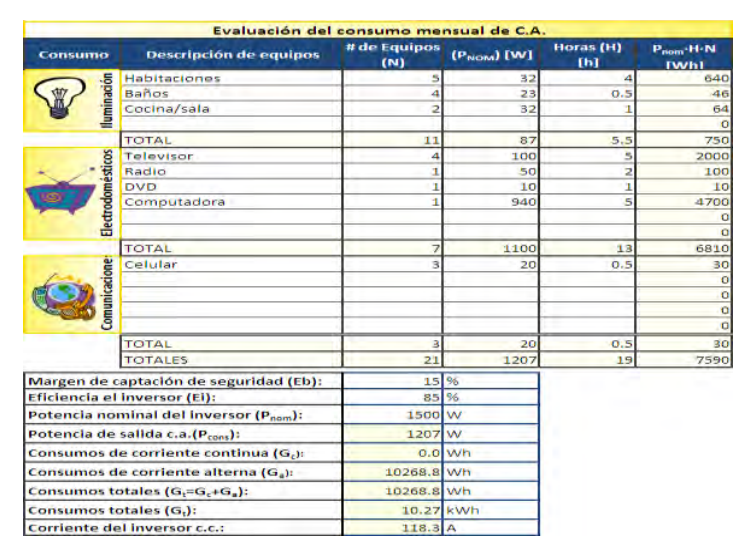

Figura №01. Cálculo de demanda energética para una vivienda.

Cuadro №03. Determinación del costo del s/./kwh del SHEFV para el periocio fébrero-marzo 2010

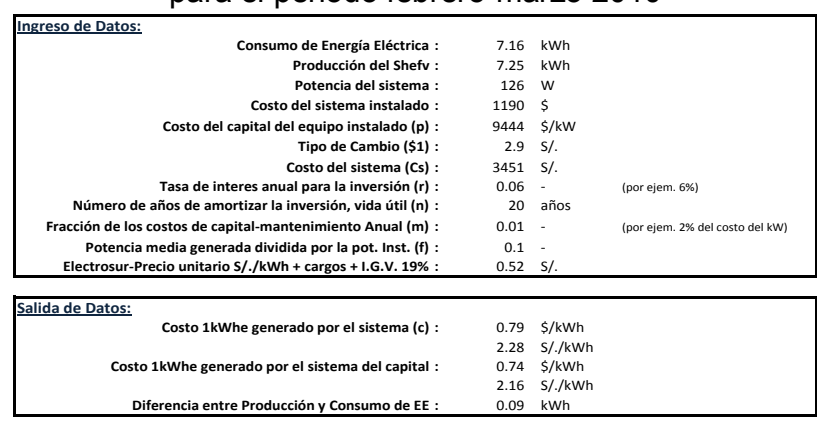

Cuadro №04. Software-plantilla SHEFV studio (beta)

\begin{tabular}{|c|c|c|c|c|c|}
\hline \multicolumn{6}{|c|}{ MENU DEL SHEFV } \\
\hline Meses & \multicolumn{5}{|c|}{ Semanas } \\
\hline Enero & 1ra & $2 \mathrm{da}$ & 3 ra & 4ta & 5 ta \\
\hline Febrero & $\underline{1 \text { ra }}$ & $\underline{2 \mathrm{da}}$ & $\underline{\text { 3ra }}$ & 4ta & \\
\hline Marzo & $\frac{1 \text { ra }}{1 \text { ra }}$ & $\frac{2 \mathrm{da} a}{2 \mathrm{da}}$ & $\frac{3 \mathrm{ra}}{3 \mathrm{~m}}$ & $\frac{4 \text { ta }}{4 \text { ta }}$ & $\frac{5 \text { ta }}{5 \mathrm{ta}}$ \\
\hline Abril & 1ra & $2 \mathrm{da}$ & $3 r a$ & 4ta & 5 ta \\
\hline Mayo & 1ra & $2 \mathrm{da}$ & 3ra & 4ta & 5 ta \\
\hline Junio & 1ra & $2 \mathrm{da}$ & 3ra & 4ta & 5 ta \\
\hline Julio & 1ra & $2 \mathrm{da}$ & 3ra & 4ta & 5 ta \\
\hline Agosto & 1ra & $2 \mathrm{da}$ & 3ra & 4ta & 5 ta \\
\hline Septiembre & 1ra & $2 \mathrm{da}$ & 3ra & 4ta & 5 ta \\
\hline octubre & 1ra & $2 \mathrm{da}$ & 3ra & 4ta & 5 ta \\
\hline Noviembre & 1ra & $2 \mathrm{da}$ & 3ra & 4ta & 5 ta \\
\hline Diciembre & 1ra & $2 \mathrm{da}$ & $3 \mathrm{ra}$ & 4ta & $5 \mathrm{ta}$ \\
\hline
\end{tabular}

\section{Comentario}

El Software-plantilla Shefv Studio (Beta) es un software que forma parte de la tesis: "Evaluación energética comparativa de un sistema híbrido eólicofotovoltaico de baja potencia para la electrificación de una vivienda rural", fue desarrollado con la finalidad de permitir el mayor control (acceder, analizar, crear y navegar) sobre 12 variables (17280 datos) con intervalos de tiempo de 1 minuto, comprendidas entre datos meteorológicos y energéticos del sistema híbrido eólico fotovoltaico que se ingresan diariamente; así también muestra los resultados obtenidos para 1 día(s), semana(s), mes(es) y/o año. El fácil diseño del software-plantilla permite construirlo en un tiempo corto y aprovechar los comandos y funciones disponibles del excel, sin perder la buena presentación de datos.

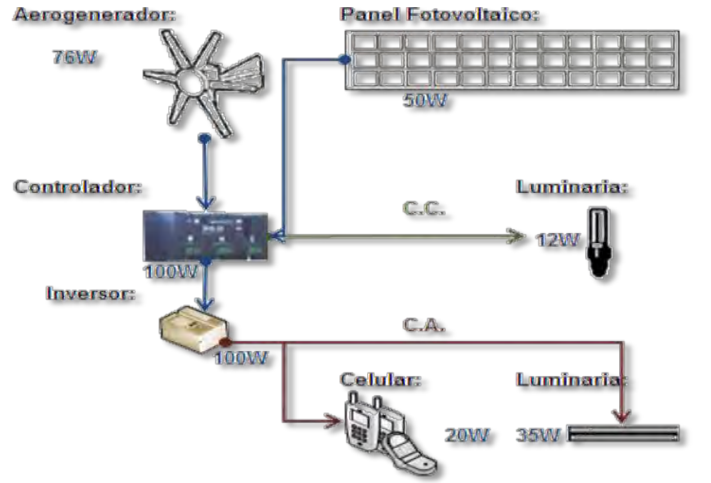

Figura №02. Esquema de la instalación del SHEFV

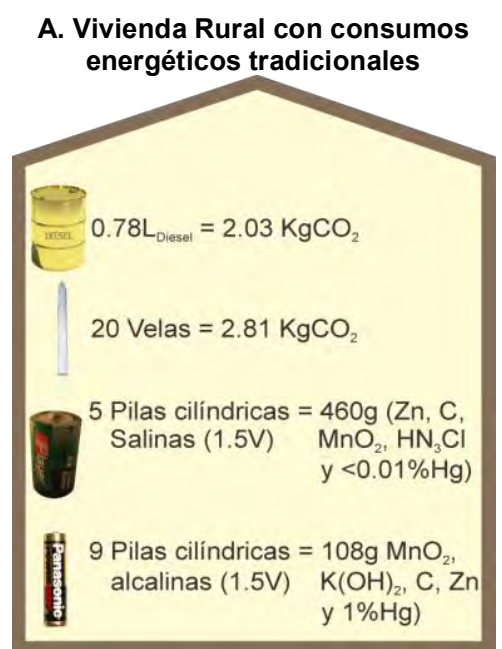

B. Vivienda Rural con Shefv

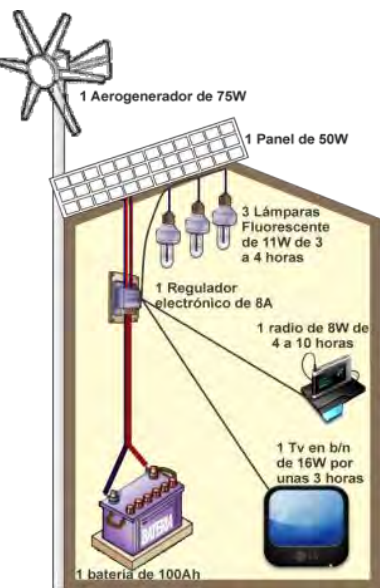

FIGURA №03. RECONVERSIÓN ENERGÉTICA DE UNA VIVIENDA RURAL

La Figura 03.A muestra una vivienda rural con consumos energéticos tradicionales mensuales, con una emisión al aire de $4.84 \mathrm{Kg}$ de Dióxido de Carbono(CO2) y con $568 \mathrm{~g}$ de elementos químicos altamente contaminantes conformados por Cinc metálico(Zn), Carbono(C), Dióxido de Manganeso(MnO2), Cloruro de Amonio(HN3Cl), Hidróxido de Potasio $(\mathrm{K}(\mathrm{OH}) 2)$ y Mercurio $(\mathrm{Hg})$; los cuales contaminarían 1'590,000 litros de agua. La Figura 03.B muestra una vivienda rural con Shefv, el cual suministra energía eléctrica para 3 lámparas fluorescentes de $11 \mathrm{~W}, 1$ radio de $8 \mathrm{~W}$ y $1 \mathrm{Tv}$ b/n de $16 \mathrm{~W}$. 


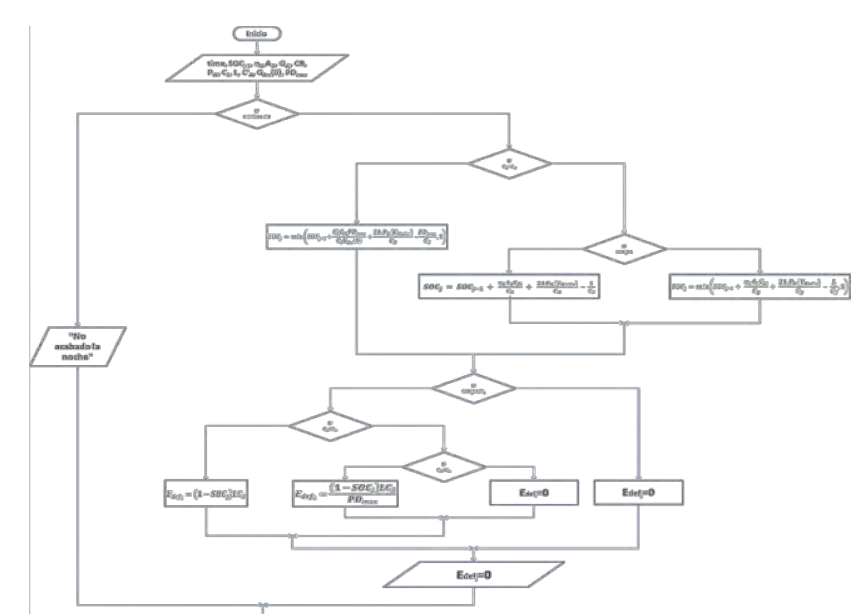

Figura №04. Diagrama de Flujo del Método numérico para el cálculo de la LLP

\section{Comentario}

El diagrama de flujo muestra los datos que se van a ingresar para el método de simulación, el cual permite calcular la LLP y la relación de la terna de valores $\left[C^{\prime} A_{v}, C S, C_{A_{w}}\right.$. Se parte de un sistema que tiene todo el consumo durante la noche, el acumulador no tiene pérdidas y la capacidad útil es nominal. Utilizando el método de simulación se calculará el $\mathrm{SOC}_{\mathrm{j}}$ (State of Charge) indicando si se necesita energía deficitaria que viene hacer la energía auxiliar. El resultado es cuánta energía se necesita o no se necesita.

\section{CONCLUSIONES}

1. Los resultados de las evaluaciones hechas a los recursos eólico y solar para la provincia de Tacna del periodo Febrero-Marzo 2010, en energía producible Solar y Eólica son: 346.32 $\mathrm{kWh} / \mathrm{m}^{2} \cdot$ mes y $4.66 \mathrm{kWh} / \mathrm{m}^{2} \cdot \mathrm{mes}$, por lo tanto la Energía producible solar es mucho mayor que la Energía producible eólica.

2. De la evaluación de la calidad final del Shefv de baja potencia para el periodo Febrero-Marzo 2010; se encontró que su eficiencia energética es de $85.7 \%$, la producción de energía eléctrica de los sistemas con sus respectivos porcentajes de aporte: Shefv: 14.5kWh (100\%), Fotovoltaico: 12.91 kWh (96\%) y Eólico: 0.55 kWh (4\%); así también el suministro de energía eléctrica promedio mensual del shefv es de $7.25 \mathrm{kWh}$ y el consumo mensual de la vivienda rural es de $7.16 \mathrm{kWh}$ indicando suministro sin déficit de energía eléctrica, se encontró que el costo del $1 \mathrm{kWh}$ generado por el Shefv es de $0.79 \$ / \mathrm{kWh}$.

3. Se realizó la reconversión energética hacia energías más limpias, para una vivienda de la provincia de Tacna con consumos energéticos tradicionales (diesel, velas y pilas) a Shefv, brindando comodidad en iluminación, sanidad y limpieza; evitando la contaminación ambiental (atmósfera y subsuelo) con $\mathrm{CO} 2$ y residuos peligrosos como: Cinc metálico(Zn), Carbono(C), Dióxido de Manganeso(MnO2), Cloruro de Amonio( $\mathrm{HN} 3 \mathrm{Cl})$, Hidróxido de Potasio $(\mathrm{K}(\mathrm{OH}) 2)$ y Mercurio( $\mathrm{Hg})$; el primero provocaría: efecto invernadero, calentamiento global, destrucción de la capa de ozono $(4.84 \mathrm{Kg}$ de Dióxido de Carbono emitidos mensualmente) y enfermedades respiratorias y ojos; el segundo liberaría al medio ambiente sustancias químicas que serían absorbidas por el subsuelo, filtrándose en los mantos acuíferos (1'590,000 litros de agua contaminada mensualmente), entrando en la cadena alimenticia trayendo consecuencias graves para la salud de las personas (retraso, perturbaciones mentales y emocionales, irritación de las vías respiratorias, ojos, piel, aumento de la presión, vómito, diarrea, pérdida de la memoria, entre otras enfermedades).

\section{RECOMENDACIONES}

1. Se debe incrementar el número de estaciones meteorológicas a sotavento debido a que la dirección del viento no es constante durante todo el año, para poder realizar futuras investigaciones como son las investigaciones en la energía eólica marina, los offshore serían una buena solución futura en la producción de energía eléctrica para Tacna, así también en la detección de zonas sensibles a la contaminación.

2. Realizar la comparación de resultados obtenidos de hojas de cálculo y softwares estadísticos, es muy importante para obtener el mejor resultado (optimizar) para la investigación.

3. La naturaleza de la investigación en ocasiones conlleva a la elaboración de un software producto de la investigación, esta herramienta nos ayudará a acceder, procesar y analizar más rápido los datos con los que se está trabajando; la ventaja del software-plantilla en comparación con los softwares radica en la rapidez y facilidad de desarrollarlo mientras se está realizando la investigación.

4. Se obtiene una mejor correlación utilizando la aproximación polinómica de orden cuatro que la aproximación potencial, para la relación experimental entre potencia y velocidad de viento. 


\section{REFERENCIAS}

[1] DEVORE J., Probabilidad y Estadística para Ingeniería y Ciencias, $6^{a}$ Ed., International Thomson Editores S.A. de C.V., México, 2005.

[2] PACCO K., Informe de prácticas preprofesionales; Vientos de Tacna (2005).

[3] TORRES H., Evaluación Energética del Potencial Energético Solar, (1997) 50.

[4] ZORA F., TACNA HISTORIA Y FOLKLORE, Editorial y librería "SANTA MARÍA", 1969.

[5] Microsoft Corporation, Microsoft $®$ Encarta $₫ 2009$ [DVD], (2008).

[6] Escudero P., Sistema mixto eólico fotovoltaico en una vivienda rural basada en el concepto de Permacultura, (Mayo 1998),(Paper), en: www.it46.se/entry/61

[7] Davis Instruments, Vantage Pro2, (Manual), en: http://www.weathershop.com/davis_vantage_pro2 .htm

[8] Fresenius W. y otros, Squirrel 1200 Data Logger Squirrel Analysis Program, (Manual de instrucción), legacy.library.ucsf.edu/tid/mcm56e00

[9] Chiroque J., Microaerogeneradores de 100W para uso rural, (Paper), en:

http://www.itdg.org.pe/publicaciones/pdf/Microaer ogenerador\%20IT\%20PE\%20100\%20para\%20ele ctrificacion\%20rural.pdf

[10] Microsiervos.com, ¿Qué es la emisión de CO2 por kilómetro recorrido? $(\mathrm{gCO} 2 / \mathrm{km})$, en: http://eco.microsiervos.com/practico/que-esemision-co2-kilometro-recorrido.html

[11] Pilasmasbaratas.com, Pila cilíndrica salina LR20 / D - 1,5V - Player, en:

http://www.pilasmasbaratas.com/pilas-salinas-Ir3Ir6-Ir20-Ir14/pila-salina-Ir20-d-1-5v-player-182.html

[12] UPME, ANTEPROYECTO DE NORMA AEROGENERADORES REQUISITOS DE SEGURIDAD, (Paper), en:

http://www.si3ea.gov.co/si3ea/documentos/docum entacion/energias alternativas/normalizacion/ANT EPROYECTO_DE_NORMA_AEROGENERADOR ES_REQUISITOS_DE_SEGURIDA.pdf

E-mail: Cleophatra_Bvza@yahoo.es 